Kansas State University Libraries

New Prairie Press

\title{
NEAREST NEIGHBOR ADJUSTED BEST LINEAR UNBIASED PREDICTION IN FIELD EXPERIMENTS
}

Walter W. Stroup

Follow this and additional works at: https://newprairiepress.org/agstatconference

Part of the Agriculture Commons, and the Applied Statistics Commons

\section{(c) (1) $\Theta($}

This work is licensed under a Creative Commons Attribution-Noncommercial-No Derivative Works 4.0 License.

\section{Recommended Citation}

Stroup, Walter W. (1990). "NEAREST NEIGHBOR ADJUSTED BEST LINEAR UNBIASED PREDICTION IN FIELD EXPERIMENTS," Conference on Applied Statistics in Agriculture. https://doi.org/10.4148/ 2475-7772.1442

This is brought to you for free and open access by the Conferences at New Prairie Press. It has been accepted for inclusion in Conference on Applied Statistics in Agriculture by an authorized administrator of New Prairie Press. For more information, please contact cads@k-state.edu. 


\title{
NEAREST NEIGHBOR ADJUSTED BEST LINEAR UNBIASED PREDICTION IN FIELD EXPERIMENTS
}

\author{
Walter $W$. Stroup \\ Department of Biometry, University of Nebraska, Lincoln, NE 68583-0712
}

Abstract: In field experiments with large numbers of treatments, inference can be affected by 1) local variation, and 2) method of analysis.

The standard approach to local, or spatial, variation in the design of experiments is blocking. While the randomized complete block design is obviously unsuitable for experiments with large numbers of treatments, incomplete block designs - even apparently well-chosen ones - may be only partial solutions. Various nearest neighbor adjustment procedures are an alternative approach to spatial variation.

Treatment effects are usually estimated using standard linear model methods. That is, linear unbiased estimates are obtained using ordinary least squares or, for example when nearest neighbor adjustments are used, generalized least squares. This follows from regarding treatment as a fixed effect. However, when there are large numbers of treatments, regarding treatment as a random effect and obtaining best linear unbiased predictors (BLUP) can improve precision.

Nearest neighbor methods and BLUP have had largely parallel development. The purpose of this paper is to put them together.

Key Words: linear model, fixed effect, random effect, generalized least squares, best linear unbiased prediction, nearest neighbor, spatial correlation.

\section{INTRODUCTION}

Experiments with large numbers of treatments are often of interest in agricultural research. For example, a plant breeder may wish to screen new cultivars or varieties; it is rare for such an experiment to have fewer than a dozen varieties and one having as many as a hundred or more varieties would not be unusual. Such trials may continue over two or more growing seasons with some varieties deleted and others added as the study progresses. For example, many states have variety-yield testing programs which proceed on this basis.

In this paper, two issues of vital concern in experiments with large numbers of treatments will be considered: local - or spatial - variation and best linear unbiased prediction (BLUP).

BLUP as an alternative to conventional best linear unbiased estimation (BLUE) has been used with great success in animal breeding studies involving large numbers of treatments (e.g. sires) and unbalanced data. Recently, Hill and Rosenberger (1985) have found that BLUP's advantages apply as well to long-term plant variety evaluation. Such work suggests 
that BLUP's advantages may be generally applicable to field experiments with large numbers of treatments.

The problem of local variability in experiments with large numbers of treatments is perhaps more widely appreciated. Nearest neighbor adjustment (NNA) methods, one aspect of which focuses on the problem of local gradients, have received a great deal of attention in recent years.

BLUP and NNA have to date seen largely parallel development. The purpose of this paper is to bring them together. The next section will briefly review important background. Then, a nearest-neighbor BLUP will be developed and some comparisons between it and other methods currently in the literature will be presented.

\subsection{BEST LINEAR UNBIASED PREDICTION}

The basis for analysis of variance, regression, and other methods which dominate current statistical practice in agriculture is the mixed linear model, whose general form is

$$
\mathrm{Y}=\mathrm{XB}+\mathrm{ZU}+\mathrm{E},
$$

where $Y$ is a vector of observations, $B$ is a vector of fixed effects, $X$ is a matrix of known constants determined by the design (treatment and/or regression) with respect to the fixed effects, $U$ is a vector of random effects, $Z$ is a the design matrix with respect to the random effects, and $E$ is a vector of residuals. $U$ and $E$ are uncorrelated, $E(U)=E(E)=0$, $\operatorname{Var}(U)=G$ and $\operatorname{Var}(E)=R$. Thus, $E(Y)=X B$ and $\operatorname{Var}(Y)=V=Z G Z^{\prime}+R$.

It is instructive to consider how fixed and random effects have been dealt with in "statistical tradition". Statistical methods texts have typically distinguished between them along these lines:

\section{FIXED EFFECT}

Specific levels included in experiment as a result of deliberate choice.

Inference is based on estimable functions of the form $\mathrm{K}^{\prime} \mathrm{B}$ (e.g. treatment means, differences, contrasts, etc.).

\section{RANDOM EFFECT}

Specific levels included in experiment as a result of random sample of target population.

Inference is based on variance or covariance components of $G$ and $R$ or functions of these components (e.g. heritability).

Despite cases for which it is not obvious whether an effect is fixed or random, in conventional statistical practice the distinction between the two types of effects is quite rigid: if one is interested in treatment means or difference, then, by definition, the effect is fixed. The fixed effect vector is estimated (optimally) via generalized least squares by solving the equation

$$
\mathrm{b}=\left(\mathrm{X}^{\prime} \mathrm{V}^{-1} \mathrm{X}\right)^{--} \mathrm{X}^{\prime} \mathrm{V}^{-1} \mathrm{Y}
$$


and the function $K^{\prime} b$ is the best linear unbiased estimator (BLUE) of $K^{\prime} B$, assuming K'B estimable (see Searle, 1971).

However, an alternative form of inference with the mixed model is the best linear unbiased predictor (BLUP) of the predictable function $\mathrm{K}^{\prime} \mathrm{B}+$ $M^{\prime} U$, where $K^{\prime} B$ is estimable. The BLUP can be obtained by solving the mixed models equations as discussed by Henderson (1975) and Harville (1976):

$$
\left[\begin{array}{l}
b \\
u
\end{array}\right]=\left[\begin{array}{cc}
X^{\prime} R^{-1} X & X^{\prime} R^{-1} Z \\
Z^{\prime} R^{-1} X & Z^{\prime} R^{-1} Z+G^{-1}
\end{array}\right]\left[\begin{array}{l}
X^{\prime} R^{-1} Y \\
Z^{\prime} R^{-1} Y
\end{array}\right]
$$

Using this solution, $K^{\prime} b+M^{\prime} u$ is the BLUP of $K^{\prime} B+M^{\prime} U$.

From this perspective, even if the objective is to estimate treatment means or differences, it does not automaticaliy follow that the effect in question is fixed. It may be more desirable to regard it as random and obtain a BLUP than to regard it as fixed and obtain a BLUE. The dilemma for the data analyst is when? Although a precise answer is not available and more work admittedly is required, a provisional answer seems to be as follows. When there are relatively few treatment levels, the conventional approach to estimating fixed effects via BLUE is fine. However, if the number of levels is large (say, 20 or more) and the distribution of the treatment effects is reasonably symmetric then BLUP appears to be better. This is what Hill and Rosenberger (1985) found and is confirmed by the simulation study discussed below.

Intuitively, the difference between BLUP and BLUE results from BLUP's use of the distribution among the treatment effects, as expressed by relevant elements of $\operatorname{Var}(U)=G$, to shrink extreme observations toward the mean. When there are many treatments and relatively few replications, some treatments will inevitably have extreme high or low estimated effects. BLUE has no provision for attenuating them; BLUP uses the treatment effect distribution. When there are fewer treatments, the likelihood of extreme treatment effect estimates is not nearly as great; the attenuation is BLUP is not as important, and the relative imprecision of the estimate of $G$ makes the quality of attenuation poor. Thus, with few treatment levels, BLUE would be preferred.

\subsection{NEAREST NEIGHBOR METHODS}

Regardless of whether BLUP or BLUE are used, neither will result in accurate inference in the presence of strong, undealt with, local gradients. For example, consider the graph given in Figure 1 . This is from a plant breeding experiment conducted at the Aridoculture Center in Settat, Morocco. The experiment involved 24 varieties of wheat and was conducted in 3 randomized complete blocks. Each block was a linear strip. Figure 1 contains the residuals for the 24 plots in the first block; the other blocks show similar patterns. Obviously, a strong, non-random trend is present. Figure 1 is a excellent illustration of a "local gradient"; such patterns are not unusual in field experiments. 
Three basic approaches to the local gradient problem are 1) incomplete block designs, 2) nearest neighbor adjustments, and 3) spatial covariance models.

Incomplete block designs are well-established in statistical literature and good discussions on them can be found in any experimental design text. A major advantage of these designs is that they can be analyzed using standard methods, e.g. SAS-GLM. A minor disadvantage is that balanced or partially balanced incomplete block designs may not exist or be easy to construct for the number of treatments, replications per treatment, and block size required by the researcher. However, computer software is being developed to select "approximately balanced" designs for most applications; while these are not true BIB or PBIB designs - a fact which makes some practitioners nervous - they are usually reasonable designs and are clearly preferable to the existing alternatives, namely an $\mathrm{RCBD}$ or nothing! A major disadvantage of incomplete block designs is the fact that it is often unclear HOW to block, that is, which sets of experimental units really are homogeneous. Uniformity trials are often suggested, but this can be rather glib advice: in many cases such a trial is not feasible; in other cases, the local gradients may vary from growing season to growing season as a result of sub-surface dynamics, rendering the uniformity trial not merely useless but severely misleading.

There are a variety of nearest neighbor methods. Papadakis (1937) presented the first widely used method. Many articles have appeared in recent years, e.g. Bartlett (1978), Wilkinson, et. al. (1983), Besag and Kempton (1985), Gleeson and Cullis (1987), Cullis, et. al. (1989). As Gleeson and Cullis (1987) note, nearest neighbor adjustment (NNA) methods all the following common approach. The linear model can be denoted as

$$
Y=X_{1} A+X_{2} T+S+E
$$

where $T$ is a vector of treatment effects, $X_{2}$ is the design matrix for treatment, $A$ is a vector of other effects such as block, location, etc., $\mathrm{X}_{1}$ is its design matrix, $S$ is a "smooth trend" (e.g. the patterned variation over plots in Figure 1 ), and $E$ is random residual variation have variance $I \sigma^{2}$. The form of $\mathrm{S}$ may be described, e.g. using some spatial covariance process such as an ARMA or ARIMA model, but the premise of NNA methods is that $S$ is too complex to be efficiently estimated with the data available. Thus, the data are re-expressed in terms of local differences in order to simplify the form of $\operatorname{Var}(S+E)$. This re-expression is done using a difference operator $D$, discussed immediately below.

The " $1^{\text {st }}$ difference operator" is a very commonly used form of $D$. Consider an experiment with $t$ treatments conducted in randomized complete blocks. A block from this design can be visualized as

$$
\begin{array}{|l|l|l|l|l|l|}
\hline T_{(1)} & T_{(2)} & \ldots & T_{(t-1)} & T_{(t)} \\
\hline
\end{array}
$$

where $T_{(i)}$ represents the $i^{\text {th }}$ treatment in spatial order in the block. Letting $y_{(i)}$ be the observation associated with $T_{(i)}$ then the first 
difference among the observations is defined as

$$
\begin{aligned}
& d_{1}=y_{(2)}-y_{(1)} \\
& d_{2}=y_{(3)}-y_{(2)} \\
& d_{t-1}=y_{(t)}-y_{(t-1)} .
\end{aligned}
$$

Thus, the difference matrix for the $j^{\text {th }}$ block is

$$
D_{j}=\left[\begin{array}{rrrrrrr}
1 & -1 & 0 & . & . & . & 0 \\
0 & 1 & -1 & . & . & \cdot & 0 \\
0 & 0 & \cdot & \cdot & \cdot & 1 & -1
\end{array}\right]
$$

and the difference matrix is

$$
D=\left[\begin{array}{ccccc}
D_{1} & 0 & \cdot & \cdot & 0 \\
0 & D_{2} & \cdot & \cdot & 0 \\
0 & 0 & \cdot & \cdot & D_{r}
\end{array}\right],
$$

where $r$ is the number of blocks. Another difference operator typically used in nearest neighbor analysis is the second difference, given by

$$
\begin{aligned}
& d_{1}=y_{(2)}-\frac{3}{2}\left[y_{(1)}+y_{(3)}\right] \\
& d_{2}=y_{(3)}-3 / 2\left[y_{(2)}+y_{(4)}\right] \\
& d_{t-2}=y_{(t-1)}-\frac{3}{2}\left[y_{(t-2)}+y_{(t)}\right] .
\end{aligned}
$$

The form of $D$ for the $2^{\text {nd }}$ difference (also called "centered first difference") follows analogously to above. In theory, any difference operator can be defined. While those discussed above only involve onedimensional arrangements of treatments, forms of $D$ for two-dimensional arrangements have considered. See, for example, Wilkinson, et. al. (1983).

Applying the difference matrix $D$ to the nearest neighbor model yields the re-expressed model

or

$$
D Y=D X_{1} A+D X_{2} T+D S+D E
$$

$$
\mathrm{d}=\mathrm{WT}+\mathrm{F}+\epsilon,
$$

where $d=D Y, W=D x_{2}, F=D S$, and $\epsilon=D e$. Note that, because of the nature of the differencing operation, which is applied within blocks, $\mathrm{DX}_{1} \mathrm{~A}=0$. Although $\operatorname{Var}(F)$ may have any form, the general purpose of the differencing operation is to simplify the "smooth trend." Typically, the approximation $\operatorname{Var}(F)=I_{n} \sigma_{f}^{2}$, where $n=\operatorname{rank}(D)$, is used. Thus, $\operatorname{Var}(d)=\operatorname{Var}(F)+\operatorname{Var}(\epsilon)$ $=I_{n} \sigma_{f}^{2}+D D^{\prime} \sigma^{2}$. Besag and Kempton note that in many instances, $\sigma^{2}$ is 
negligible relative to $\sigma_{f}^{2}$; if so, $\operatorname{Var}(d) \approx I_{n} \sigma_{f}^{2}$.

The BLUE of estimable functions $K^{\prime} T$ can be obtained using the GLS solution for the vector of treatment effects:

$$
t=\left(W^{\prime}[\operatorname{Var}(d)]^{-1} W\right)^{-} W^{\prime}[\operatorname{Var}(d)]^{-1} d,
$$

where $t$ is the estimate of $T$. Note that in obtaining the NNA BLUE, $T$ is implicitly a vector of fixed effects.

To date, all nearest neighbor methods in the literature obtain BLUE's of the treatment effects. Gleeson and Cullis (1987) obtain BLUP's on the systematic trend, i.e. within-block gradient effects. Cullis, et. al. (1989) obtain BLUP's for "standard" plant varieties, but obtain BLUE's for all remaining treatment effects. However, no comprehensive nearest neighbor method for obtaining BLUP's has been reported.

To obtain the NNA BLUE, $T$ will be considered a vector of random effects such that $E(T)=0$ and $\operatorname{Var}(T)=G$. Typically, $G=I_{t} \sigma_{\tau}{ }^{2}$. The BLUP for $T$ can now be obtained solving the mixed model equations. For the NNA model, the difference matrix eliminates the fixed effects component of the model. Thus, only the lower right-hand quadrant remains. Solving, the NNA-BLUP of $\mathrm{T}$ is

$$
t=\left(W^{\prime}[\operatorname{Var}(d)]^{-1} W+G^{-1}\right)^{-} W^{\prime}[\operatorname{Var}(d)]^{-1} d
$$

The primary difference between NNA BLUP and BLUE is that BLUP utilizes information regarding variation among treatment effects contained in $G$.

While NNA works well enough in many cases, there are objections. Among these are 1) it is clumsy to apply in two-dimensional cases; 2) it is not clear how to handle "border plots"; 3) it is not clear how to handle missing data; and 4) it is not clear that simple differences adequately address many complex spatial patterns. Indeed, these issues, especially 2) through 4), frequently are dealt with in practice by seemingly arbitrary rules of thumb. An alternative to NNA is to model the local gradients directly by including spatial covariance components in $\operatorname{Var}(\mathrm{Y})$, especially in $R$.

One alternative is to model spatial covariance using ARMA or ARIMA models. This has been discussed by Gleeson and Cullis (1987). Another is to use models from geostatistics. These, often referred to as "kriging," have been discussed by Journel and Huijbregts (1978) and have seen wide application in many disciplines, but not as yet in the analysis of field experiments. However, in principle, such application is not difficult.

There are many "kriging" models. All model the covariance between observations as a non-increasing function of distance. That is, two observations close together would be highly correlated, whereas two more distant observations would be less correlated, or uncorrelated. For example, in the "spherical" model 


$$
R=\left[\begin{array}{llllll}
I_{11} & I_{12} & \cdots & \cdot & \cdot & I_{10} \\
& I_{22} & \cdot & \cdot & \cdot & I_{20} \\
& & \cdot & & \\
& & & \cdot & \\
& & & & I_{n n}
\end{array}\right]
$$

where $r_{i 1}=\sigma^{2}$

$$
\begin{aligned}
& r_{i j}=\sigma^{2}\left[1-(3 h / 2 c)+\left(h^{3} / 2 c^{3}\right)\right] \text { if } h<c \text {, where } h \text { is the } \\
& \text { distance between the } i^{\text {th }} \text { and } j^{\text {th }} \\
& r_{i j}=0 \text { of } h \geq c
\end{aligned}
$$

Using this covariance model, the GLS equation (1) or mixed model equation (2) can be solved to obtain BLUE or BLUP, respectively, of estimable or predictable functions of $T$, the vector of treatment effects, depending on whether treatments are considered fixed or random. If the components of $R$ are unknown, they can be estimated using maximum likelihood (ML) or restricted maximum likelihood (REML). Here, two components or $\mathrm{R}$ must be estimated, $\sigma^{2}$ and $c$. Taking the vector of $1^{\text {st }}$ derivatives of the likelihood function of $Y$ with respect to $\sigma^{2}$ and $c$ respectively and the matrix of expected values of $2^{\text {nd }}$ derivatives, the Fisher Scoring algorithm can be used to obtain ML or REML estimators. This method is adapted from Harville (1977). An example is given in section 4.

\section{NNA BLUP VS. NNA BLUE AND NON-NNA ALTERNATIVES - SIMULATION STUDY}

To evaluate NNA BLUP versus alternative methods, a simulation study was performed. The alternative methods were NNA BLUE, incomplete block BLUE, incomplete block BLUP, and randomized complete block BIUE. For the NNA methods, both $1^{\text {st }}$ and $2^{\text {nd }}$ difference operators were using and $\operatorname{Var}(d)$ was modeled with $\sigma^{2} \approx 0$ and with $\sigma^{2}>0$. Since the differences between BLUP and BLUE are most likely to be important in unbalanced data, 2 unbalanced simulations were performed. The first, called the "2-year experiment," used 30 treatments, 20 observed in the "first year" with 2 complete block replications; 10 treatments were dropped and 10 new treatments added for observation in the "second year." The second simulation, called the "3year experiment," was similar except that 24 treatments were used. 12 treatments were observed, replicated in 2 complete blocks, during the "first year," 6 were dropped and 6 new treatments added for the second year, and likewise for the third year. For each simulated experiment, treatment means were estimated (or predicted, in the cases involving BLUP). MSE, correlation, and rank correlation of the estimated/predicted versus actual means were calculated for each case.

Each set of complete blocks was structured so that it could also be viewed as an incomplete block design. That is, each complete block could alternatively be partitioned into several incomplete blocks and analyzed accordingly. For example, in the "2-year experiment," a typical arrangement was: 


\begin{tabular}{l|llll|llll|lllll|llllll|l|llll|}
$\mathrm{R} 1$ & 1 & 2 & 3 & 4 & 5 & 6 & 7 & 8 & 9 & 10 & 11 & 12 & 13 & 14 & 15 & 16 & 17 & 18 & 19 & 20 \\
\hline R2 & 5 & 9 & 13 & 17 & 1 & 10 & 14 & 18 & 2 & 6 & 15 & 19 & 3 & 7 & 11 & 20 & 4 & 8 & 12 & 16
\end{tabular}

where R1 and R2 denote the two complete blocks and the divisions within the blocks signify incomplete blocks. This particular arrangement represents 2 replications of a 4 × 5 rectangular lattice.

In the "3-year experiment," a representative arrangement was:

R2 \begin{tabular}{|llll|llll|lllll|}
\hline 1 & 2 & 3 & 4 & 5 & 6 & 7 & 8 & 9 & 10 & 11 & 12 \\
\hline 5 & 9 & 1 & 12 & 2 & 6 & 8 & 10 & 3 & 4 & 7 & 11
\end{tabular}

The local gradient was simulated with $\sigma_{f}^{2}=2, \sigma^{2}=1$, and $\sigma_{\tau}^{2}=2$. This was used because it produced a local gradient large enough relative to treatment variance to affect inference if not accounted for without being unrealistically large and because it produced local gradients similar to those actually observed in the field. Figure 2 gives an example of a simulated gradient. Note its similarity in form to the data from Morocco in Figure 1.

The results are given in Table 1 . The main findings can be summarized as follows. First, using MSE as a criterion, BLUP was consistently more precise than BLUE. Second, the nearest neighbor methods, were consistently more precise than the incomplete block alternatives. Third, among the nearest neighbor methods, estimating $\sigma^{2}$ rather than setting $\sigma^{2} \approx 0$ did not improve the precision - this confirms the validity of the Besag and Kempton's (1985) simplification and is good news for users, since computing requirements are sharply reduced when $\sigma^{2} \approx 0$. Fourth, among nearest neighbor methods, the $1^{\text {st }}$ difference method resulted in greater precision than the $2^{\text {nd }}$ difference method. While this may be a consequence of the way local gradients were simulated, the fact that the simulated gradients were similar to those observed in field data suggests that simple difference operators are adequate in practice. Fifth, when NO local gradient was present, the $1^{\text {st }}$ difference NNA-BLUP compared favorably to non-NNA methods, suggesting that its use will not "hurt" anything, even when not needed. Finally, when spatial variability Is present, the results obtained using the standard RCBD approach are catastrophic.

\section{A "SPHERICAL KRIGING" EXAMPLE}

Figure 3 describes data from a hypothetical experiment whose error structure is described by the "spherical kriging" covariance matrix given in (3). The data are arranged in an 8 plot by 8 plot grid. Imagine that these data result from a uniformity trial and that the objective is to evaluate 16 treatments. Thus, 4 replications are possible. A superficial look at the contour plot might suggest dividing the "field" into 4 squares and assigning treatments using a randomized complete block design typically, this is what field researchers will in fact do. Upon closer inspection, the plots within the 4 squares are not really homogeneous; a 
more careful researcher might partition these squares into rows (say - or columns) and assign the treatments using, for example, a $4 \times 4$ balanced lattice design. An even more effective approach would use the gradients to define blocks, even though the result may be non-rectangular, possibly discontinuous blocks. Most field researchers would be reluctant to actually do this. There are many reasons for this reluctance, some legitimate, some not. Given this reality, in this comparison the latter blocking scheme will not be considered a viable alternative.

Table 2 contains the results of these two approaches compared with the method of calculating REML estimates of $\sigma^{2}$ and the range parameter described in equation (3) and then using the estimate of $R$ to compute GLS estimates of the treatment effects. In this case, BLUE was used rather than BLUP, since the data are balanced. Note that this method, referred to in Table 2 as the "spherical kriging" method, results in much more accurate inference, both in terms of a closer match between the "true" and estimated treatment effects and in terms of reduced standard error of treatment differences. This is typical of several simulated data sets with the same or similar spatial covariance parameters.

Obviously, more study is needed, including a simulation study of adequate size and scope. However even this limited study does indicate that 1) the parameters of covariance models suggested by geostatistics can indeed be estimated using standard variance component methods (e.g. REML), 2) the estimation of treatment effects in the presence of such spatial correlation can thus be integrated into standard mixed linear model methodology, and 3 ) such estimators appear to be more precise than those obtained using standard analysis of blocked designs.

\section{SUMMARY AND CONCLUSIONS}

In experiments with large numbers of treatments, unaccounted for spatial variability can dramatically decrease the precision of estimated treatment effects. Both NNA methods using difference operators to simplify the covariance structure and direct estimation of the covariance parameters using REML appear to be effective in accounting for spatial variability. For unbalanced experiments, e.g. long-term studies, classical estimation (BLUE) results in less efficient estimates of treatment effects than BLUP. In such experiments, treatment effects seem to behave more like random than fixed effects and thus should be modelled accordingly.

There are several areas for further study. All the methods described warrant further investigation; their properties under many conditions in which they might be used are not fully understood. There may be alternatives to the REML and ML algorithms used in this paper that are preferable in certain cases. More systematic study of "best case" and "worst case" performance of these methods is needed. Appropriate confidence interval and hypothesis testing procedures are not well developed for any but the simplest NNA methods. Kackar and Harville (1984) have discussed approximate procedures for the general mixed model, but these need to be adapted to the cases discussed in this paper. Finally, most of the methods discussed here require prodigious computer resources (e.g. time and memory). Efficient computing algorithms need to be developed and software useable to data analysts needs to be developed. 


\section{References}

1. Bartlett, M.S. (1978) Nearest neighbor models in the analysis of field experiments (with discussion). Journal of the Royal Statistical Society, Series B. 40: 147-174.

2. Besag, J.E. and Kempton, R.A. (1986) Statistical analysis of field experiments using neighboring plots. Biometrics 42: 231-251.

3. Cullis, B.R., Lill, W.J., Fisher, J.A., Read, B.J., and Gleeson, A.C. (1989) A new procedure for the analysis of early generation variety trials. Applied Statistics. 38: 361-376.

4. Gleeson, A.C. and Cullis, B.R. (1987) Residual maximum likelihood (REML) estimation of a neighbor model for field experiments. Biometrics 43: 277-288.

5. Harville, D.A. (1976) Extension of the Gauss-Markov theorem to include the estimation of random effects. Annals of Statistics. 4: 384-395.

6. Harville, D.A. (1977) Maximum likelihood approaches to variance component estimation and related problems. Journal of the American Statistical Association 72: 320-338.

7. Henderson, C.R. (1975) Best linear unbiased prediction under a selection model. Biometrics 31: 423-447.

8. Hill, R.R. and Rosenberger, J.L. (1985) Methods for combining data from germplasm evaluation trials. Crop Science 25: 467-470.

9. Journel, A.G. and Huijbregts, C.J. (1978) Mining Geostatistics. Academic Press. London, UK.

10. Kackar, R.N. and Harville, D.A. (1984) Approximations for standard errors of estimators of fixed and random effects in mixed linear models. Journal of the American Statistical Association. 79:853-862.

11. Papadakis, J.S. (1937) Methode statistique pour des experiences sur champ. Bulletin de l'Institut d'Amelioration des plantes. Thessalonike 23.

12. SAS Institute, Inc. (1985) SAS/IML User's Guide, Version 5 Edition. SAS Institute, Inc. Cary, NC.

13. Searle, S.R. (1971) Linear Models. John Wiley and Sons. New York.

14. Swallow, W. and Monahan, J. (1984) Simulation study comparing variance component estimators for the two-stage nested design. Technometrics 26: $47-57$.

15. Wilkinson, G.N., Eckert, S.R., Hancock, T.W., and Mayo, O. (1983) Nearest neighbor (NN) analysis of field experiments (with discussion). Journal of the Royal Statistical Society, Series B. 45: 152-212. 
Figure 1. Moroccan data: plot of residuals for each block

PLOTID denotes identification number of plot in block.

$B L O C K=1$

PLOT OF RESIDUAL $*$ PLOTID LEGEND: $\mathrm{A}=1$ OBS, $\mathrm{B}=2$ OBS, ETC.

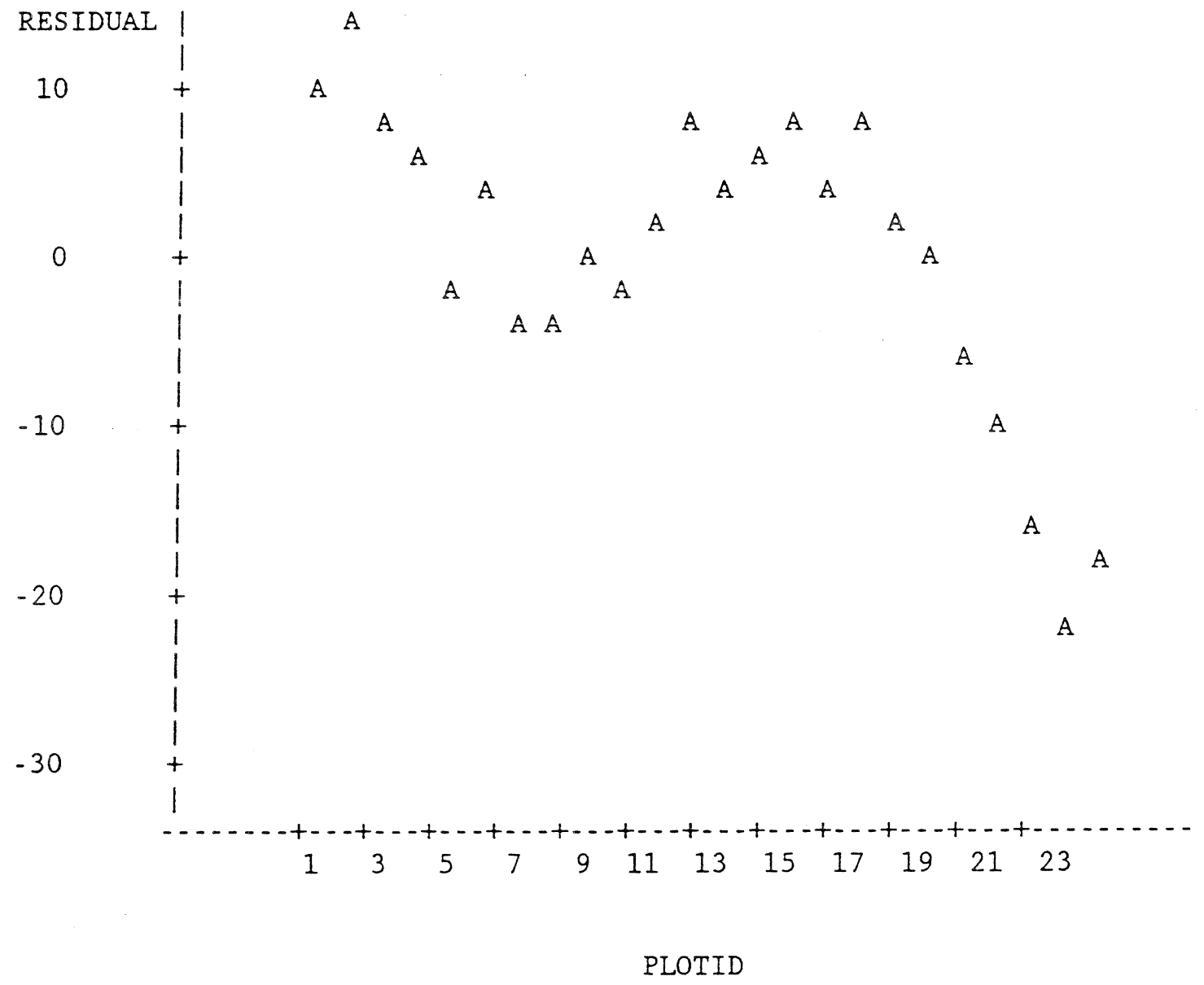


Figure 2. Example of typical nearest neighbor gradient plots from simulated data

\section{EXAMPLE 2}

PLOT OF F*PLOT LEGEND: $\mathrm{A}=1 \mathrm{OBS}, \mathrm{B}=2 \mathrm{OBS}$, ETC.

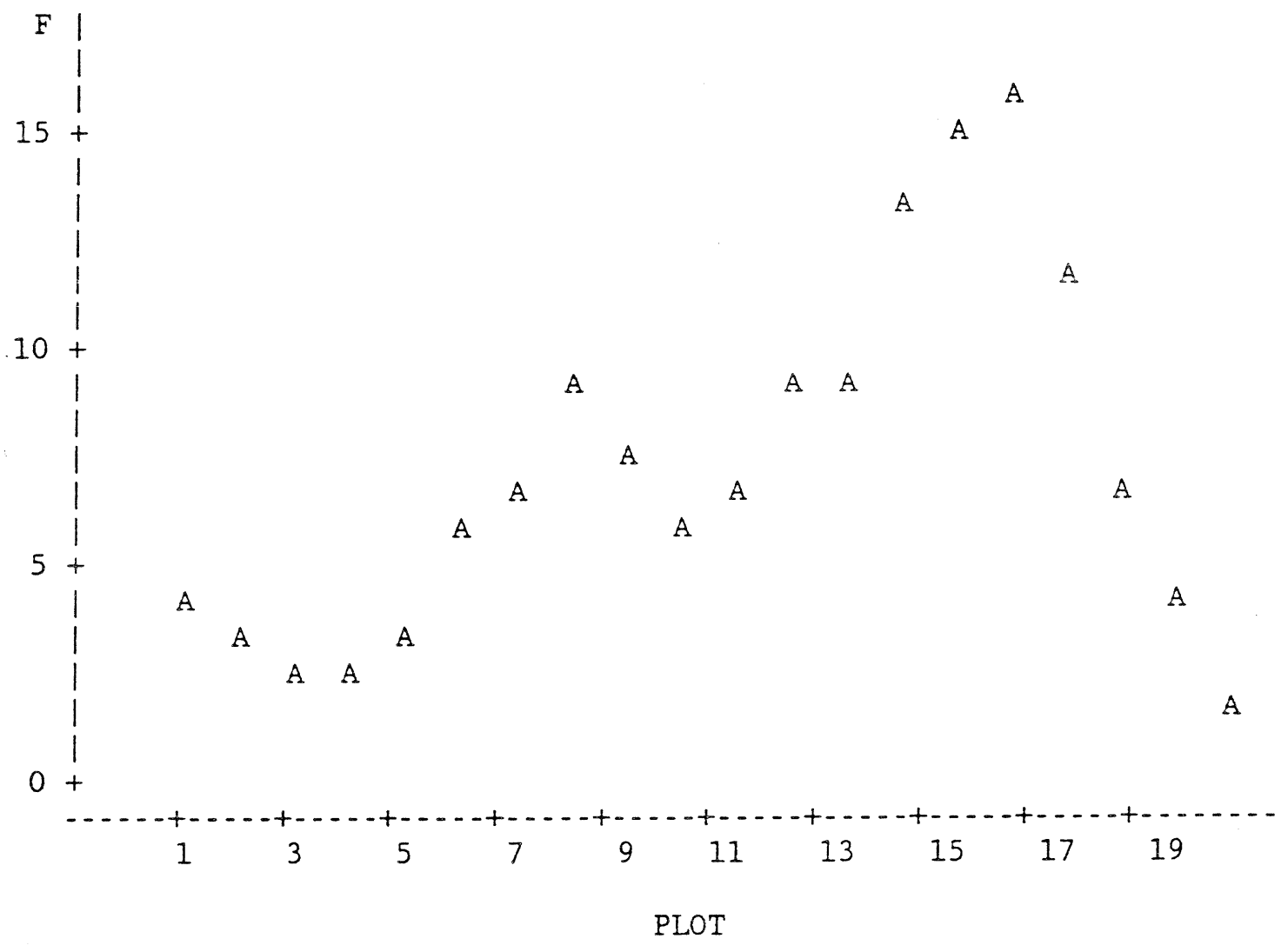


Figure 2. Contour plot for hypothetical uniformity trial from Section 4.

\begin{tabular}{|c|c|}
\hline \multicolumn{2}{|r|}{$0+1+1+1+1+W W T$} \\
\hline & $\star \star \star \star * * * * *$ WWWWWWWW00000000+1+1+1+WWWWWWWWWWWWWWWW . . \\
\hline \multirow{3}{*}{$8+$} & $* \star \star * * * * * *$ WWWWWWWW00000000+1+1+1+WWWWWWWWWWWWWW . . \\
\hline & $\star * \star * \star * * * *$ WWWWWWWW00000000+1+1+1+1+WWWWWWWWWWWWWWWW . . . . . 00000000 \\
\hline & WWWWWWWW0000000000000000+1+1+1++*********WWWWWWWWWWWWWWWW . . . . . . \\
\hline i & WWWWWWWW0000000000000000++1+1+1+********WWWWWWWWWWWWWWWW . . . . . . \\
\hline \multirow{2}{*}{$7+$} & WWWWWWWW0000000000000000+1+1+1+**********WWWWWWWWWWWWWWWW . . . . . . \\
\hline & WWWWWWWW $0000000000000000+1+1+1+* * * * * * * *$ WWWWWWWWWWWWWWWW . . . . . . \\
\hline i & WWWWWWWW00000000000000000000000000000000 \#WWWWWWWWWWWWWWWW \\
\hline I & WWWWWWWW00000000000000000000000000000000 WWWWWWWWWWWWWWWW \\
\hline \multirow{2}{*}{$6+$} & WWWWWWWW00000000000000000000000000000000 \#WWWWWWWWWWWWWWW \\
\hline & WWWWWWWW00000000000000000000000000000000 \#WWWWWWWWWWWWWW \\
\hline \multirow{2}{*}{1} & WWWWWWWW0000000000000000+1+1+1+1+**********+1+1+1+1+0000000000000000 \\
\hline & WWWWWWWW $0000000000000000+++++1++* * * * * * * * * *++++++++0000000000000000$ \\
\hline \multirow[t]{2}{*}{$5+$} & WWWWWWW $0000000000000000+++++1++* * * * * * * * * *+1+++1++0000000000000000$ \\
\hline & WWWWWWWW0000000000000000+1+1+1+1+*x*2*x*x*x*1+1+1+1+0000000000000000 \\
\hline & $+1+1+1+1+1+1+1+1+\ldots \ldots \ldots \ldots \ldots+1+1+1+1+1++++$ WWWWWWWW \\
\hline & $H+1+1+1++1+1+1+1+1+1+\ldots \ldots \ldots \ldots \ldots h++++++++++++++$ WWWWWWWW \\
\hline \multirow[t]{2}{*}{$4+$} & $+1+1+1+1+1+1+1+1+1++1+\ldots \ldots \ldots \ldots \ldots++++++++++++++$ WWWWWWWW \\
\hline & $H+1+1+1+1+1+1+1++++\ldots \ldots \ldots \ldots \ldots+1+++++++++++++$ WWWWWWWW \\
\hline \multirow[t]{2}{*}{$i$} & 00000000 WWWWWWW00000000 $+++++++++++++++\ldots \ldots .00000000+1+1++++$ \\
\hline & $00000000 W W W W W W W 00000000++1+++1+1+1++1+\ldots \ldots \ldots 00000000+1+1+1+1$ \\
\hline \multirow[t]{3}{*}{$3+$} & 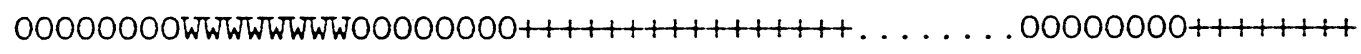 \\
\hline & 00000000WWWWWWWW00000000 $+1+1+1+1+1+1+1+\ldots \ldots \ldots 00000000+1++1++1$ \\
\hline & $0000000000000000+++1++1++1+++1++00000000$ WWWWWWWW $\ldots \ldots \ldots+++1++++$ \\
\hline & $0000000000000000+1+1++++++++++++00000000$ WWWWWWWW $\ldots \ldots \ldots++++++++$ \\
\hline \multirow[t]{2}{*}{$2+$} & $0000000000000000+1+1+1+1+1++++++00000000$ WWWWWWWW $\ldots \ldots \ldots++1+1+++$ \\
\hline & $0000000000000000+++++++++1+++++00000000$ WWWWWWWW $\ldots \ldots \ldots++++++++$ \\
\hline \multirow{2}{*}{1} & WWWWWWWWWWWWWWW0000000000000000WWWWWWWW0000000000000000. \\
\hline & WWWWWWWWWWWWWWW0000000000000000WWWWWWWW0000000000000000. \\
\hline \multirow[t]{3}{*}{$1+$} & WWWWWWWWWWWWWWWW0000000000000000WWWWWWWW0000000000000000. \\
\hline & WWWWWWWWWWWWWWWW0000000000000000WWWWWWWW0000000000000000. \\
\hline & WWWWWWWWWWWWWWWW0000000000000000WWWWWWWWO \\
\hline
\end{tabular}

0
0

\begin{tabular}{|c|c|c|c|c|c|c|c|}
\hline Symbol & RESPON & & Symbol & RESP & NSE & Symbol & RESPONSE \\
\hline & $0.00-$ & 3.75 & 00000 & 7.50 & 11.25 & $* * * * * x$ & $15.00-18.75$ \\
\hline$+111+$ & $3.75-$ & 7.50 & WWWWW & 11.25 & 15.00 & $\# \# \#$ & $18.75-22.50$ \\
\hline
\end{tabular}


Table 1. Simulation results for estimation of treatment effects.

2 YEAR EXPT - WITH NEIGHBOR EFFECT

METHOD OF ANALYSIS

1st DIFF, $\sigma^{2} \approx 0$, BLUP

Ist DIFF, $\sigma^{2} \approx 0$, BLUE

2nd DIFF, $\sigma^{2} \approx 0$, BLUP

2nd DIFF, $\sigma^{2} \approx 0$, BLUE

Ist DIFF, $\sigma^{2}>0$, BLUP

1st DIFF, $\sigma^{2}>0$, BLUE

2nd DIFF, $\sigma^{2}>0$, BLUP

2nd DIFF, $\sigma^{2}>0$, BLUE

INC BLK, BLUP

INC BLK, BLUE

RAND COMP BLK
MSE

1.349

1.830

1.573

2.107

1.376

1.863

1.561

2. 509

2.107

2.112

8.459
CORR

.822

.822

.798

.800

.823

.820

.796

.779

.741

.734

.571
RANK CORR

.797

.798

.776

.778

.798

.795

.772

.752

.716

.707

.546

3 YEAR EXPT - WITH NEIGHBOR EFFECT

METHOD OF ANALYSIS

1st DIFF, $\sigma^{2} \approx 0$, BLUP

1st DIFF, $\sigma^{2} \approx 0$, BLUE

2nd DIFF, $\sigma^{2} \approx 0$, BLUP

2nd DIFF, $\sigma^{2} \approx 0$, BLUE

1st DIFF, $\sigma^{2}>0$, BLUP

1st DIFF, $\sigma^{2}>0$, BLUE

2nd DIFF, $\sigma^{2}>0$, BLUP

2nd DIFF, $\sigma^{2}>0$, BLUE

INC BLK, BLUP

INC BLK, BLUE

RAND COMP BLK
MSE

1.252

1.860

1.571

2.384

1.255

1.825

1.518

2.805

1.769

2.794

5.658
CORR

.840

.819

.809

.783

.841

.823

.803

.762

.792

.750

.648
RANK CORR

.816

.799

.787

.765

.818

.802

.784

.745

.769

.730

.635 
Table 1 (continued)

2 YEAR EXPT - NO NEIGHBOR EFFECT

METHOD OF ANALYSIS

1st DIFF, $\sigma^{2} \approx 0$, BLUP

1st DIFF, $\sigma^{2} \approx 0$, BLUE

2nd DIFF, $\sigma^{2} \approx 0$, BLUP

2nd DIFF, $\sigma^{2} \approx 0$, BLUE

INC BLK, BLUP

INC BLK, BLUE

RAND COMP BLK
MSE

CORR

1.743

2.558

2.116

3.177

1.767

2.453

1.779
.798

.789

.759

.751

.807

.798

.844
RANK CORR

.769

.761

.730

.722

.777

.770

.815

3 YEAR EXPT - NO NEIGHBOR EFFECT

METHOD OF ANALYSIS

1st DIFF, $\sigma^{2} \approx 0$, BLUP

1st DIFF, $\sigma^{2} \approx 0$, BLUE

2nd DIFF, $\sigma^{2} \approx 0$, BLUP

2nd DIFF, $\sigma^{2} \approx 0$, BLUE

INC BLK, BLUP

INC BLK, BLUE

RAND COMP BLK
MSE

CORR

1.894

2.974

2.528

4.120

1.704

2.813

2.054
.759

.747

.717

.703

.769

.748

.810
RANK CORR

.741

.730

.702

.688

.752

.730

.784 
Table 2. Comparison of Treatment Effect Estimates and Standard Errors Produced by RCBD, Lattice, and "Spherical Kriging" Analyses in the Presence of Spatial Covariance*

\begin{tabular}{ccccr} 
Parameter & True & RCBD & Lattice & Spher \\
\cline { 1 - 3 } & & & & \\
$\tau_{1}$ & 3 & 2.22 & 2.68 & 2.94 \\
$\tau_{2}$ & 3 & 2.93 & 2.92 & 3.27 \\
$\tau_{3}$ & 2 & 0.73 & 1.05 & 1.53 \\
$\tau_{4}$ & 2 & 0.59 & 1.11 & 1.41 \\
$\tau_{5}$ & 1 & 1.40 & 1.77 & 1.11 \\
$\tau_{6}$ & 1 & 0.80 & 1.40 & 1.00 \\
$\tau_{7}$ & 0 & -1.68 & -1.13 & -0.95 \\
$\tau_{8}$ & 0 & -0.37 & -0.98 & -0.18 \\
$\tau_{9}$ & 0 & 0.64 & 1.66 & 0.51 \\
$\tau_{10}$ & 0 & 1.21 & 1.25 & 0.54 \\
$\tau_{11}$ & -1 & 0.56 & -0.95 & -0.56 \\
$\tau_{12}$ & -1 & -1.19 & -1.57 & -0.96 \\
$\tau_{13}$ & -2 & -0.69 & -1.23 & -1.61 \\
$\tau_{14}$ & -2 & -1.57 & -0.94 & -1.79 \\
$\tau_{15}$ & -3 & -2.60 & -2.49 & -2.99 \\
$\tau_{16}$ & -3 & -2.96 & -4.52 & -3.30 \\
$c^{2}$ & & & & \\
$\sigma^{2}\left(\tau_{1}-\tau_{2}\right)$ & 3 & NA & NA & 3.39 \\
s.e. & NA & 1.51 & 1.75 & 3.71 \\
& & 1.32 & 1.05 & 0.58
\end{tabular}

* Using data depicted in Figure 3 with treatment effect added.

** $\tau_{i}$ denotes $i^{\text {th }}$ treatment effect, c denotes range, $\sigma^{2}$ denotes error variance, and s.e. $\left(\tau_{1}-\tau_{2}\right)$ denotes standard error of the difference between treatments 1 and 2. NA means Not Applicable. 